\title{
Control of Temperature Using PID Controller
}

\author{
Radhika Nair ${ }^{1}$, K. R. Mohan ${ }^{2}$ \\ ${ }^{1}$ Student, Electrical and Electronics Engineering Department, AIT College, Chikkamagaluru-577102 Karnataka, India \\ ${ }^{2}$ HOD and Adjunct Faculty, Electrical and Electronics Department, AIT College, Chikkamagaluru-577102 Karnataka, India
}

\begin{abstract}
Through our project we are showing the control of constant temperature according to the desired value (set point) in a closed loop using PID controller system. For this, we are using a microcontroller, a temperature sensor for sensing the temperature of the closed loops. By using the microcontroller we compare the desired value with current value and it is displayed in the LCD. Also to provide the constant temperature, Fan or Heater is turned on or off according with the variations of current temperature in ${ }^{\circ} \mathrm{C}$ from desired setpoint.
\end{abstract}

Keywords: PID controller, Microcontroller, Sensors

\section{Introduction}

The objective of our project "TEMPERATURE PID CONTROLLLER" is maintaining the constant temperature in a particular area using PID controller.

Whatever the process or the parameter (temp, flow, speed, ..) the principles of control are similar. Input and output signals are specified in this project is digital. Control of a process is achieved by means of a closed loop circuit. This project is prepared in order to control the temperature of a furnace in the best and easiest possible way.

The control system is that means by which any quantity of interest in a machine, mechanism or other equipment is maintained or altered in accordance with a desired manner. Here we have used the closed loop system; that is the feedback system. The feedback signal is derived from the output of the system. This signal gives the capability to act as self-correcting mechanism. The beneficial effects of the feedback in the system with high loop gain. The controlled variable accurately follows the desired value and also feedback in a control system greatly improves the speed of its response.

One of the primary purposes of using feedback in control system is to reduce the sensitivity of the system to parameter variations.

The project deals with a simple aspect of giving information about the controlling of temperature in a furnace. In this project we are developing a system, which can control temperature of a furnace automatically. The system is be capable of taking decisions accordingly of overheating of blast furnace and cooling of a furnace.

This project is done by using microcontroller (PIC 16F873A) which was developed by microchip company with several features than processors with cheap cost. A temperature LM 35 is used in sensing the temperature and relays like heater or fan are used for adjusting the temperature with desired temperature value. The functions occurring are displayed on the liquid crystal display.
In this system, it can implement any applications about controlling or monitoring the temperature without any human effort.

\section{Block Diagram}

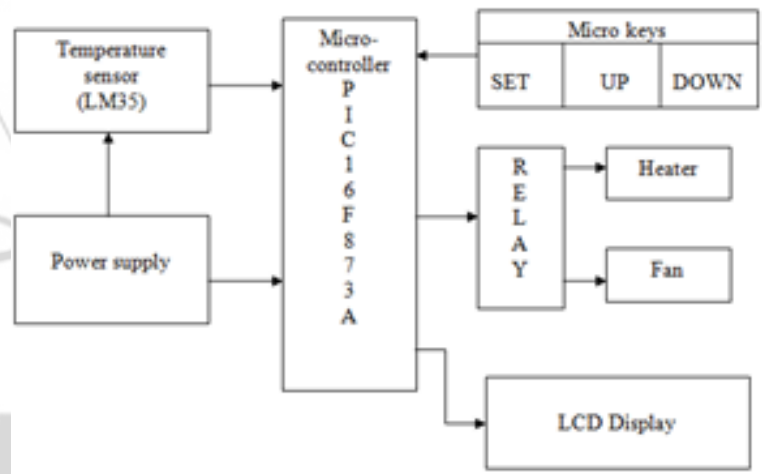

Figure 1: Block diagram for micro-controller

\subsection{Block Diagram Description}

The block diagram for "temperature PID controller" circuit consist of

- IC LM 35

- PIC 16F873A

- POWER SUPPLY

- RELAY

- DISPLAY SECTION

A fixed three terminal voltage regulator has a regulated $\mathrm{dc}$ output voltage of $5 \mathrm{v}$ and provide it to IC LM 35, PIC 16 f873A, micro keys, relays and display section

Temperature sensing section consists of an IC LM 35 which acts as a transducer. It senses the temperature and converts it into voltage as a scale of $1^{\circ} \mathrm{C}$ into $10 \mathrm{mv}$.

At the heart of the circuit is microcontroller PIC 16F873A with many advantages and it is available in RISC architecture.

The output of the microcontroller is given to the relays and display section. 


\section{International Journal of Science and Research (IJSR) \\ ISSN (Online): 2319-7064}

Index Copernicus Value (2013): 6.14 | Impact Factor (2015): 6.391

Relays we used here are Heater and Fan; they are used for adjusting the obtained temperature with the desired temperature value.

The display section, through the IC LM020L, that displays temperature. It is the main observable part of this whole system.

\subsection{Overall Circuit Diagram}
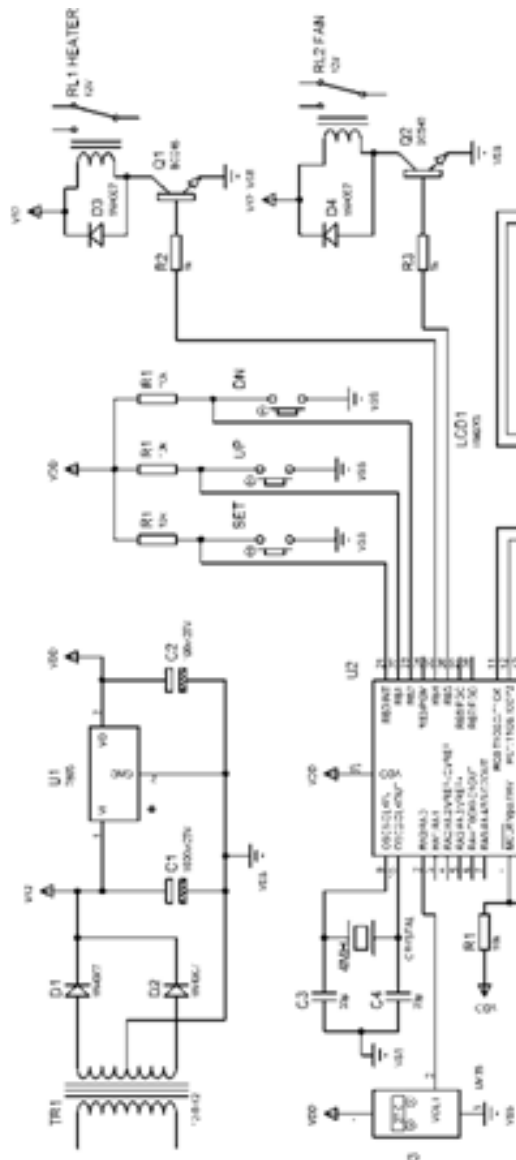

$5^{\circ} \mid+$

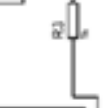

正
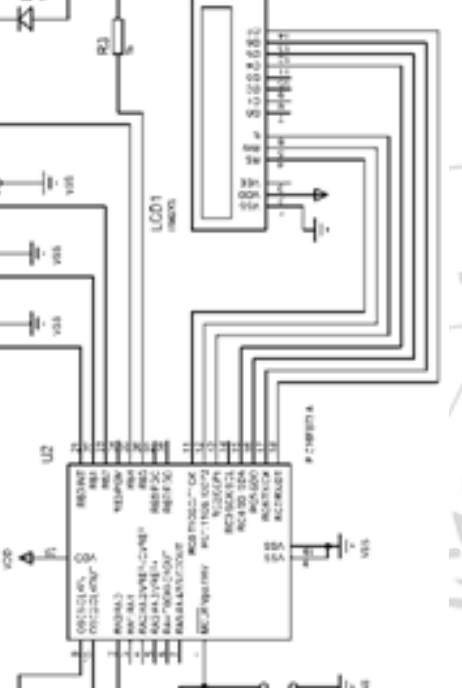

Figure 2: Circuit Diagram for the controller

\subsection{Overall Circuit Diagram Working}

The circuit shows microcontroller based temperature PID controller using temperature sensor.Microcontroller PIC $16 \mathrm{~F} 873 \mathrm{~A}$ is the heart of the circuit. It is available inRISC architecture. The PIC 16F873A is a mid-range 8-bit CPU optimized for ControlApplications. It has 35 instructions on chip flash program memory.

LM35 used as the temperature sensor. It sense the current temperature of a closed loop and converts into corresponding voltage as it is a transducer. It is connected to pin 2 (RA0/AN0) of microcontroller. The microcontroller circuit is connected with reset circuit and crystal oscillator circuit. Crystal oscillator is the one used to generate the pulses to the microcontroller and it is also called as the heart of microcontroller. Here we have used $4 \mathrm{MHz}$ crystal which generates pulses. It offers the highest precision (exactness/accuracy) and stability.

Even the microcontroller has an internal RC oscillator with a maximum frequency of $4 \mathrm{MHz}$, noise affect it easily.
Because of increasing of aging of oscillator, resonant frequency varies and cannot get the fixed frequency. So we use crystal oscillator externally for accuracy.

To set up the desired temperature value, we use the micro keys such as SET, UP, DOWN. And also the tolerance value is set in the firmware using embedded $\mathrm{C}$ language.

According to the comparison of desired temperature (here we say as 'Set point') with the current temperature, the relay - Heater or Fan is worked.

\section{Case I:}

If the current temperature is greater than desired temperature (including tolerance) then turn off the heater and turn on the fan.

\section{Case II:}

If the current temperature is less than the desired temperature (including tolerance), then turn on the heater and turn off the fan.

\section{Case III:}

Else turn off both heater and fan.The relays such as heater connected to pin 25 (RB4) and fan is connected to pin 26 (RB5).

The processing of controller will display in the LCD. The current temperature as ' $\mathrm{CT}$ ' and the set point as 'SP' can be observed on the first line of LCD. And also, the present conditions of the relays are displayed on the second line of the LCD.

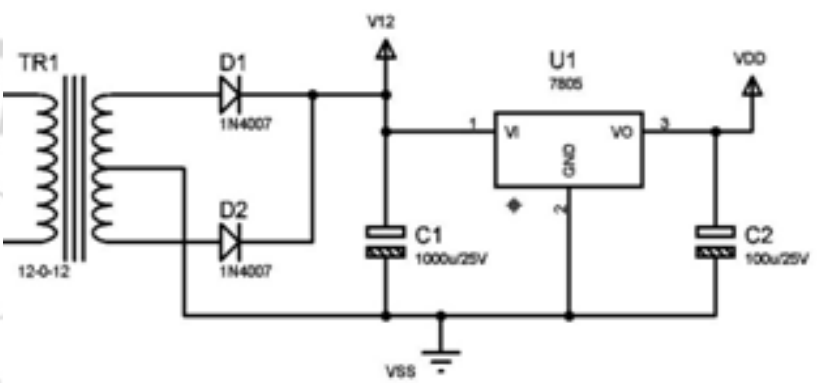

Figure 3: Circuit diagram for regulator

\section{Working Principle}

\section{Transformer}

The transformer will step down the power supply voltage $(0$ $-230 \mathrm{~V})$ to $(0-6 \mathrm{~V})$ level. Then the secondary of the potential transformer will be connected to the center-tapped full-wave rectifier; where diodes are working in the property of one-side conduction capability.

\section{Center tap-Rectifier}

In a rectifier, a center-tapped transformer and two diodescan form a full-wave rectifier that allows both half-cycles of the $\mathrm{AC}$ waveform to contribute to the direct current, making it smoother than a half-wave rectifier. A center-tapped rectifier is preferred to the full bridge rectifier when the output DC current is high and the output voltage is low. The advantages of using precision rectifier are it will give peak voltage output as dc; rest of the circuits will give only RMS output. 


\section{International Journal of Science and Research (IJSR) \\ ISSN (Online): 2319-7064}

Index Copernicus Value (2013): 6.14 | Impact Factor (2015): 6.391

\section{FILTERS}

Pre-filter and post-filter are connected to the regulator IC. Distance between pre-filter and post-filter should be $5 \mathrm{~cm}$. High frequency post-filters are used.

\section{IC VOLTAGE REGULATOR}

Voltage regulators comprise a class of widely used ICs. Regulator IC units contain the circuitry for reference source, comparator amplifier, control device, and overload protection all in a single IC. IC units provide regulation of either a fixed positive voltage, a fixed negative voltage, or an adjustable set voltage. The regulators can be selected for operation with load currents from hundreds of milliamperes to tens of amperes, corresponding to power ratings from milli watts to tens of watts.

A fixed three terminal voltage regulator has an unregulated $\mathrm{dc}$ input voltage, $\mathrm{Vi}$, applied to one input terminal, a regulated dc output voltage, Vo, from a second terminal, with the third terminal connected to ground.

The series 78 regulators provide fixed positive regulated voltages from 5 to 24 volts.

- For ICs, microcontroller, LCD --------- 5volts.

- For relay circuits ------------ 12volts.

\section{Microcontroller Circuit}

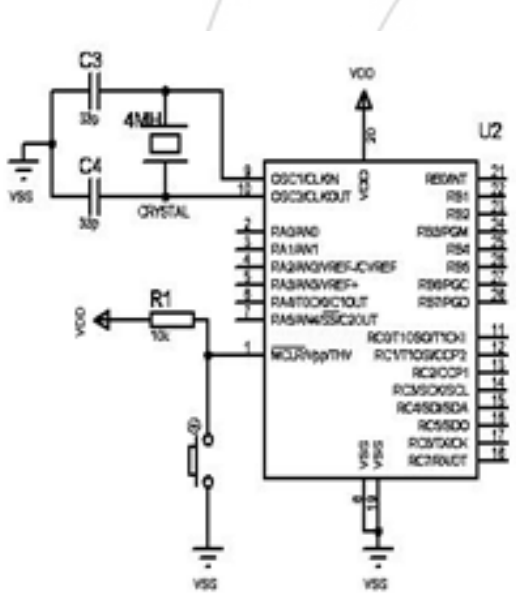

Figure 4: Circuit diagram for microcontroller.

The microcontroller circuit is connected with reset circuit, crystal oscillator, LCD circuit; the reset circuit is the one which is an external interrupt which is designed to reset the program. And the crystal oscillator circuit is the one used to generate the pulses to the microcontroller and it also called as the heart of the microcontroller.

The Liquid Crystal Display which is used to display the what we need the LCD has fourteen pins in which three pins for the command and eight pins for the data. If the data is given to LCD it is write command which is configured by the programmer otherwise it is read command in which data read to microcontroller the data pins are given to the port 0 and command pins are given to the port 2 .

Other than these pins a one pin configured for the contrast of the LCD. Thus the microcontroller circuit works.
A microcontroller is a complete microprocessor built on a single IC. Microcontrollers were developed to meet a need for microprocessors to be put into low cost products.

To solve the problem in microprocessor system is implemented with a single chip microcontroller. This could be called microcomputer, as all the major part are in the IC. Most frequently they are called microcontroller because they are used to perform control functions.

The microcontroller contains full implementation of a standard MICROPROCESSOR, ROM, RAM, I/O, CLOCK, TIMERS, and also SERIAL PORTS. Microcontroller also called "system on a chip" or "single chip microprocessor system" or "computer on a chip".

Another term to describe a microcontroller is embedded controller, because the microcontroller and its supports circuits are often built into or embedded in the devices they control.

\section{Advantages}

- If "a system is developed with a microprocessor, the designer has to go for external memory such as RAM, ROM or EPROM and peripherals and hence the size of the PCB will be large enough to hold all the required peripherals. But, the microcontroller has got all these on a single chip so development of a similar system with a microcontroller reduces PCB size and cost of the design.

- One of the major differences between a microcontroller and microprocessor is that a controller often deals with bits, not bytes as in the real world application.

- It has only 35 instructions, so it is easy to learn.

- Design complexity is small.

- It has eight level stacks. And also addresses are in vectored form (pre-defined).

\section{Applications}

A microcontroller is a kind of miniature computer that you can find in all kinds of Gizmos. Some examples of common, every-day products that have microcontrollers are built in. if it has buttons and a digital display, chances are it also has a programmable microcontroller brain.

Microcontrollers are designed for use in sophisticated real time applications such as

1) Industrial Control

2) Instrumentation and

3) Intelligent computer peripherals

They are used in industrial applications to control

- Motor

- Robotics

- Discrete and continuous process control

- In missile guidance and control

- In medical instrumentation

- Oscilloscopes

- Telecommunication

- Automobiles

- For scanning a keyboard

- Driving an LCD 


\section{International Journal of Science and Research (IJSR) \\ ISSN (Online): 2319-7064}

Index Copernicus Value (2013): 6.14 | Impact Factor (2015): 6.391

- For frequency measurements

- Period measurements

- Machinery

- Aerospace designs

- And other high tech devices

\section{Result}

The below figure shows the output of various controllers and it gives the output of the circuit.

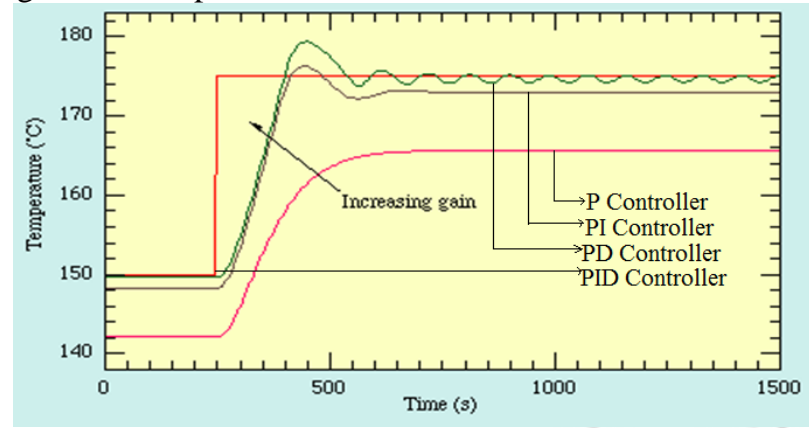

Figure 5: Waveform of PID controller

\section{Conclusion}

We are pleasure to conclude our project on the topic "Temperature PID Controller". The detail of this project has been made by members of our team sincerely with the inspiration of out tutors.

Through this project we have done, sensing and maintaining the temperature using IC LM35, which is very sensitive. This IC is ideal for interfacing with microcontroller PIC16F873A.

According to the program installed in the PIC, it regulates or works the relays based on the comparison of current temperature with set point. And the working of the system can be observed in the LCD.

\section{Future Scope}

Now-a-days also these types of circuits can be used in large farms and in certain places where we want to measure the current temperature and also to maintain the temperature constant according to the user's decision.

We can use in a wide variety of applications like:

- Poultry farm

- Industries

- Thermal furnace

- Boiler

- Medical applications

- And in all temperature controlling areas

\section{References}

[1] J.G. Ziegler and Nichols, "Optimal Settings for Automatic Controllers", Trans. ASME, vol. 64 P.P. 759$768,1942$.
[2] K.J. Astrom and T. Haughland, "Automatic Tuning of PID Controllers", $1^{\text {st }}$ ed. Research Triangle Park, NC:Instrum.Soc. Amer, 1988.

[3] K.J. Astrom and T. Haughland, "Automatic Tuning of PID Controllers", $1^{\text {st }}$ d. Research Triangle Park, NC:Instrum. Soc. Amer, 1995.

[4] K. Ogata, "Modern Control Engineering", $3{ }^{\text {rd }}$ ed. Upper saddle River, NJ: Prentice-Hall 1997.

[5] J.C. Basilio and S.R. Matos, "Design of PI and PID Controllers with Transient Performance Specification",IEEE Transactions on Education, Vol. 45, No. 4, November 2002.

[6] Salim Ahmed, Biao Huang, SirishL. shah, " Novel identification Method from Step Response", ControlEngineering Practice 15 (2007), 545550, Science Direct. 